\title{
Predicting survival after pulmonary metastasectomy for colorectal cancer: previous liver metastases matter
}

\author{
Ulrich Landes' ${ }^{1}$, John Robert 1 , Thomas Perneger², Gilles Mentha' ${ }^{1}$ Vincent Ott 1 , Philippe Morel ${ }^{1}$ and Pascal Gervaz ${ }^{* 1}$
}

\begin{abstract}
Background: Few patients with lung metastases from colorectal cancer (CRC) are candidates for surgical therapy with a curative intent, and it is currently impossible to identify those who may benefit the most from thoracotomy. The aim of this study was to determine the impact of various parameters on survival after pulmonary metastasectomy for CRC.

Methods: We performed a retrospective analysis of 40 consecutive patients (median age 63.5 [range 33-82] years) who underwent resection of pulmonary metastases from CRC in our institution from 1996 to 2009.

Results: Median follow-up was 33 (range 4-139) months. Twenty-four (60\%) patients did not have previous liver metastases before undergoing lung surgery. Median disease-free interval between primary colorectal tumor and development of lung metastases was 32.5 months. 3- and 5-year overall survival after thoracotomy was $70.1 \%$ and $43.4 \%$, respectively. In multivariate analysis, the following parameters were correlated with tumor recurrence after thoracotomy; a history of previous liver metastases ( $\mathrm{HR}=3.8,95 \% \mathrm{Cl} 1.4-9.8)$; and lung surgery other than wedge resection ( $\mathrm{HR}=3.0,95 \% \mathrm{Cl} 1.1-7.8$ ). Prior resection of liver metastases was also correlated with an increased risk of death $(\mathrm{HR}=5.1,95 \% \mathrm{Cl} 1.1-24.8, \mathrm{p}=0.04)$. Median survival after thoracotomy was 87 (range 34-139) months in the group of patients without liver metastases versus 40 (range 28-51) months in patients who had undergone prior hepatectomy $(p=0.09)$.
\end{abstract}

Conclusion: The main parameter associated with poor outcome after lung resection of CRC metastases is a history of liver metastases.

\section{Background}

Resection of hepatic metastases from colorectal cancer $(\mathrm{CRC})$ has yielded 5 -year survival rates ranging from $25 \%$ to $50 \%[1,2]$. Similarly, resection of lung metastases from CRC has yielded 5-year survival rates ranging from $20 \%$ up to $60 \%$ in large series [3,4]. Based on these encouraging results, many surgeons have expanded the indications for resecting metastatic CRC, and there is nowadays growing pressure to perform lung metastasectomy, even in asymptomatic CRC patients. The issue therefore is to select the patients with pulmonary metastases who are good candidate for surgical therapy with a curative intent. Unfortunately, it is currently not possible to do so - hence the necessity for surgeons to preoperatively identify clin-

* Correspondence: pascal.gervaz@hcuge.ch

1 Department of Surgery, Geneva University Hospital and Medical School, Geneva, Switzerland

Full list of author information is available at the end of the article ico-pathological parameters predicting survival after thoracotomy.

LM develop in 5-15\% of CRC patients according to two different scenarios: the first scenario, most common, is the metachronous development of lung metastases in a patient who has previously developed in transit liver metastases; in the second scenario - less frequent patients develop lung metastases synchronous or metachronous to primary colorectal cancer, but without evidence of liver metastases ("skip metastases") [5]. In the latter situation, the reason why the liver does not provide an adequate soil for the metastases to develop is unclear, but might involve, among other factors, deficient tumor angiogenesis [6]. Most CRC patients included in surgical series of pulmonary metastasectomy belonged to the second category. 
Since 2000, about 20 series have investigated the outcome of CRC patients who underwent resection of lung metastases with a curative intent. Reported 5-year overall survival rates range from $24 \%$ [7] to $68 \%$ [8], indicating that these studies reflect the experience of highly specialized centres with a selected subset of patients [9]. Various factors associated with prolonged survival after surgery for lung metastases from CRC have been identified, including: a) a long disease-free interval (defined as the time from colectomy to the development of lung metastases [10-12]; b) prethoracotomy carcinoembryogenic antigen (CEA) level <5NG/ML [13-15]; c) a single isolated metastasis $<3 \mathrm{~cm}$ in size [16-18]; and d) the absence of thoracic lymph node invasion $[19,20]$. By contrast, a history of previous liver metastases has never been documented as statistically significant. However, many series of pulmonary metastasectomy for CRC included mostly patients without liver metastases, or were conducted at a time when hepatectomy was rarely considered in patients with extensive liver metastases. The present study was conducted in order to assess the impact of prior surgery for liver metastases on the outcome of CRC patients who subsequently underwent pulmonary metastasectomy.

\section{Methods}

We performed a retrospective analysis of all CRC patients who underwent thoracotomy for lung metastases with a curative intent in our institution since 1996. Lung surgery was performed in the Thoracic Surgery Unit and both resections of primary tumor and liver metastases were performed in the Visceral Surgery Unit of Geneva University Hospital. We included all patients with a histopathological diagnosis of colorectal adenocarcinoma metastatic to the lung, whether or not they underwent prior liver surgery. The following parameters were recorded and considered for statistical analysis: 1) patients' characteristics; age, gender: 2) primary tumor characteristics; TNM stage; location (colon or rectum); preoperative radiation therapy; adjuvant chemo-and/or chemotherapy: 3 ) characteristics of liver metastases when present; number of metastases and location (uni- versus bilobar); size of largest metastases: adjuvant chemotherapy; and type of surgery (metastasectomy versus segmentectomy versus right/left hepatectomy; 4) characteristics of lung metastases: number and size of the largest metastases; type of surgery performed (wedge versus lobectomy versus pneumonectomy); presence of involved mediastinal lymph nodes; adjuvant chemotherapy; disease-free interval between primary tumor resection and development of liver/lung metastases; and location of lung metastases (uni-versus bilateral). The study was conducted in accordance with institutional guidelines of the Ethics Committee for Clinical Research of Geneva University Hospital.
Eligibility for surgical resection of lung metastases from CRC was based upon four criteria: 1) control of primary tumour considered as achieved; 2) absence of extrathoracic lesions at the time of lung surgery; 3 ) possibility to perform a complete resection (R0) of pulmonary metastases; and 4) adequate pulmonary reserve to tolerate the planned resection, in accordance with classic oncologic criteria for pulmonary metastasectomy. Follow-up was performed in the Surgical Oncology Unit, with repeated clinical examination and thoracoabdominal CT scan imaging once a year for 5 years to detect local or systemic tumor recurrence. In addition all patients underwent colonoscopy surveillance at 1,3 and 5 years post colectomy.

\section{Statistical analysis}

Survival was assessed from the time of thoracotomy to the time of last follow-up, and statistical analysis was performed using SPSS ${ }^{\circ}$ software version 17.0 (SPSS, Chicago, Illinois, USA). Continuous variables were collapsed into clinically meaningful categories and compared using chisquare $^{2}$ tests. Logistic regression was used to identify variables associated with either tumor recurrence or death. We considered for the multivariate model all variables with univariate $\mathrm{p}$-values $<0.20$, and retained in the final model all variables with an adjusted $\mathrm{p}$-value $<0.1$. Survival curves were calculated according to the KaplanMeier method and difference between groups were analyzed with log-rank test. All tests were two-sided and a p value $<.05$ was considered statistically significant.

\section{Results}

Between February 1996 and July 2009, forty patients (median age 63.5 [range 33-82] years) underwent resection of pulmonary metastases from CRC. Median followup was 33 (range 4-139) months. Twenty-four (60\%) patients did not have previous liver metastases before undergoing lung surgery. Sixteen patients underwent resection of liver metastases with a curative intent. Median disease-free interval between liver surgery and the development of lung metastases was 17 (range 0-60) months. Median disease-free interval between primary colorectal tumor and development of lung metastases was 32.5 (range 0-82) months. 27 (67\%) patients presented with a single lung metastasis, and 24 (60\%) of lung metastases were $<3 \mathrm{~cm}$ in size. Prethoracotomy CEA serum level was missing in 22 patients and was elevated in 11 of the remaining 18 patients. This parameter was therefore not considered for inclusion in statistical analysis. The clinical and pathological characteristics of these 40 patients and their tumors are summarized in Table 1.

There were no postoperative deaths. For the whole group, 3- and 5-year overall survival rates after thoracotomy were 70.1 and $43.4 \%$, respectively (Figure 1 ). In uni- 
Table 1: Clinical and Pathological characteristics of $40 \mathrm{crc}$ patients who underwent lung metastasectomy

\begin{tabular}{|c|c|}
\hline Gender (F/M) & $13 / 27$ \\
\hline Age (median, range) years & $63.5(33-82)$ \\
\hline \multicolumn{2}{|l|}{ Primary Tumor T stage } \\
\hline 1 & 3 \\
\hline 2 & 14 \\
\hline 3 & 16 \\
\hline 4 & 7 \\
\hline \multicolumn{2}{|l|}{ Liver metastases } \\
\hline Yes/No & $16 / 24$ \\
\hline $\begin{array}{l}\text { Liver metastases Number (median, } \\
\text { range) }\end{array}$ & $2(1-8)$ \\
\hline \multicolumn{2}{|l|}{ Liver metastases Location } \\
\hline Unilobar (right/left) & $9 / 4$ \\
\hline Multilobar & 3 \\
\hline \multicolumn{2}{|l|}{ Lung metastases Number } \\
\hline Single & 27 \\
\hline Multiple & 13 \\
\hline \multicolumn{2}{|l|}{ Lung metastases Size } \\
\hline$<3 \mathrm{~cm}$ & 24 \\
\hline$>3 \mathrm{~cm}$ & 16 \\
\hline \multicolumn{2}{|l|}{ Type of lung resection } \\
\hline Wedge resection or segmentectomy & 32 \\
\hline Lobectomy & 7 \\
\hline Pneumonectomy & 1 \\
\hline $\begin{array}{l}\text { Disease-free interval median (range) } \\
\text { months }\end{array}$ & $32.5(0-82)$ \\
\hline \multicolumn{2}{|l|}{ Colectomy to lung metastases } \\
\hline $\begin{array}{l}\text { Disease-free interval median (range) } \\
\text { months }\end{array}$ & $17(0-60)$ \\
\hline Hepatectomy to lung metastases & \\
\hline
\end{tabular}

variate analysis, the presence of synchronous liver and/or lung metastases in the primary tumor (M1/stage IV) was associated with a high risk for tumor recurrence after thoracotomy (HR $=3.1 ; 95 \%$ Confidence Interval 1.2-7.8, $\mathrm{p}=0.01$ ) (Table 2). In multivariate analysis, two parameters were correlated with tumor recurrence after thoracotomy; 1 ) a history of previously resected liver metastases $(\mathrm{HR}=3.8,95 \% \mathrm{CI} 1.4-9.8)$; and 2) lung surgery other than wedge resection $(\mathrm{HR}=3.0,95 \% \mathrm{CI} 1.1-7.8)$. Prior surgery for liver metastases was also correlated with an increased risk of death $(\mathrm{HR}=5.1,95 \% \mathrm{CI} 1.1-24.8, \mathrm{p}=0.04)$ (Table 3 ). Median survival after thoracotomy was 87 (range 34139) months in the group of patients without liver surgery versus 40 (range 28-51) months in patients who underwent prior hepatectomy for liver metastases (logrank test, $\mathrm{p}=0.09$ ). 5 -year overall survival rate was $53.8 \%$ in the group of patients with isolated lung metastases versus $29.2 \%$ in the group of patients who had undergone prior liver metastasectomy (Figure 2).

\section{Discussion}

Our series differs from most published reports focusing on pulmonary metastasectomy, because of the high (40\%) percentage of patients who underwent surgery for liver metastases prior to thoracotomy. With a median followup of 33.5 and 64 months after thoracotomy and colectomy, respectively, 17 (42.5\%) patients are alive and disease-free, with an overall 5-year survival rate of $43 \%$. The main parameter associated with both tumor recurrence $(\mathrm{HR}=3.8)$ and death $(\mathrm{HR}=5.1)$ after thoracotomy is a history of resected liver metastases. Median survival after pulmonary metastasectomy was twice longer in CRC patients without previous liver surgery (87 versus 40 months).

With recent advances in surgical technique as well as adjuvant chemotherapy, many CRC patients treated with a curative intent for liver metastases have a prolonged survival; in specialized centres (and in selected patients), 5 -year overall survival rate after hepatectomy for CRC liver metastases exceeds 50\% [21]. Unfortunately, some of them will eventually develop extra-hepatic tumor recurrence, usually in the lung and may be candidates to additional pulmonary metastasectomy; most clinicians would concur that R0 resection of pulmonary metastases may improve survival in this subset of patients [22]. Whether these patients have identical survival benefit in comparison with those patients who developed isolated lung metastases remains controversial [23]; the data presented herein, however, clearly indicates that prior surgery for liver metastases is associated with a higher risk for tumor recurrence and death after pulmonary metastasectomy. These results appear hardly surprising (patients with both liver and lung metastases are more likely to have systemic disease), yet the correlation between prior liver metastasectomy and poor survival in patients undergoing pulmonary metastasectomy had not been reported so far.

Riquet et al. reported a series of 127 patients who underwent pulmonary metastasectomy; twenty-nine (23\%) of them had undergone previous liver surgery for liver metastases. Overall 5-year survival was similar to ours (41\%), but there was a trend towards better survival in patients who had undergone previous resection of liver metastases [24]. In addition, five recent series (800 patients in total) have failed to identify prior liver metastases as a risk factor for poor outcome after lung metastasectomy in CRC patients $[8,16,19,20,25]$. In the largest series of pulmonary metastasectomy for CRC, the presence of extrathoracic disease was an independent predictive factor for poor survival, but it was unclear whether these patients had prior liver surgery or not [26]. 
Table 2: Relative hazards of recurrence or death in patients with pulmonary metastases secondary to colo-rectal cancer (univariate analysis in cox proportional hazards model, time since operation for lung metastasis)

\begin{tabular}{|c|c|c|c|c|}
\hline \multirow[b]{2}{*}{ Gender ( $F$ versus $M)$} & \multicolumn{2}{|c|}{ Recurrence } & \multicolumn{2}{|c|}{ Death } \\
\hline & $2.1(0.8-5.1)$ & 0.12 & $1.7(0.6-4.4)$ & 0.30 \\
\hline Age (per 10 years) & $0.84(0.52-1.34)$ & 0.46 & $1.05(0.61-1.79)$ & 0.86 \\
\hline Primary Tumor T stage & & 0.34 & & 0.60 \\
\hline $1-2$ & 1.0 & & 1.0 & \\
\hline 3 & $4.0(0.5-30.5)$ & & $2.8(0.4-21.7)$ & \\
\hline 4 & $2.6(0.3-23.3)$ & & $3.0(0.3-27.1)$ & \\
\hline Tumor 3-4 (vs. 1-2) & $3.6(0.5-26.8)$ & 0.22 & $2.8(0.4-21.6)$ & 0.32 \\
\hline Primary Tumor N stage & & 0.23 & & 0.42 \\
\hline 0 & 1.0 & & 1.0 & \\
\hline 1 & $2.3(0.8-6.9)$ & & $1.7(0.5-5.6)$ & \\
\hline 2 & $2.7(0.8-9.3)$ & & $2.2(0.6-7.8)$ & \\
\hline Node 1-2 (versus 0) & $2.4(0.9-6.7)$ & 0.09 & $1.9(0.7-5.5)$ & 0.22 \\
\hline Primary tumors $M$ stage & $3.2(1.1-8.9)$ & 0.03 & $1.4(0.5-4.5)$ & 0.53 \\
\hline \multicolumn{5}{|l|}{ M1 (versus M0) } \\
\hline Stage 3-4 (versus 1-2) & $2.4(0.9-6.7)$ & 0.09 & $1.8(0.6-5.1)$ & 0.28 \\
\hline Liver metastasis & $3.1(1.2-7.8)$ & 0.01 & $2.3(0.8-6.2)$ & 0.10 \\
\hline Time primary to lung (per year) & $0.9(0.6-1.2)$ & 0.51 & $1.0(0.8-1.4)$ & 0.87 \\
\hline Time primary to first metastasis (per year) & $0.71(0.49-1.02)$ & 0.06 & $0.9(0.6-1.2)$ & 0.45 \\
\hline Multiple versus single lung metastasis & $1.2(0.5-3.3)$ & 0.67 & $1.2(0.9-1.7)$ & 0.17 \\
\hline Size lung metastasis $>20 \mathrm{~mm}$ & $1.8(0.7-4.8)$ & 0.21 & $1.2(0.4-3.2)$ & 0.76 \\
\hline Neo-adjuvant treatment for lung metastasis & $2.8(0.6-13.0)$ & 0.20 & $3.4(0.9-12.4)$ & 0.06 \\
\hline Adjuvant treatment for lung metastasis & $0.5(0.1-3.9)$ & 0.52 & $1.1(0.3-4.9)$ & 0.89 \\
\hline $\begin{array}{l}\text { Lung operation other than wedge (versus } \\
\text { wedge) }\end{array}$ & $2.3(0.9-5.9)$ & 0.06 & $1.8(0.7-5.0)$ & 0.23 \\
\hline
\end{tabular}

Our study has several limitations, mostly related to the small number of patients and the fact that perioperative management of patients was not uniform in terms of (neo) adjuvant chemotherapy. There is certainly a possibility for introducing selecting bias in non-randomized studies. The strength of our series is related to the high percentage of patients who underwent prior liver surgery, allowing for sound comparison of survival between groups with adequate statistical power despite limited number of events. Finally, this series reflects the outcome of a relatively homogenous population of patients who were treated according to similar clinical guidelines, by a small group of surgeons over a relatively short period of time.

Table 3: Multivariate analysis of risk factors for tumor recurrence and death

\begin{tabular}{|c|c|c|c|c|}
\hline \multirow[b]{2}{*}{ Node 1-2 (versus 0) } & \multicolumn{2}{|c|}{ Recurrence } & \multicolumn{2}{|c|}{ Death } \\
\hline & $1.7(0.6-5.0)$ & 0.34 & $1.5(0.5-4.8)$ & 0.48 \\
\hline Time primary to first metastasis (per year) & $0.83(0.48-1.44)$ & 0.51 & $1.3(0.8-2.1)$ & 0.35 \\
\hline Liver metastasis & $2.7(0.7-10.0)$ & 0.14 & $5.1(1.1-24.8)$ & 0.04 \\
\hline Size lung metastasis $>20 \mathrm{~mm}$ & $2.1(0.6-6.6)$ & 0.23 & $0.9(0.3-2.7)$ & 0.86 \\
\hline Multiple versus single lung metastasis & $1.4(0.4-4.6)$ & 0.58 & $3.4(0.9-12.9)$ & 0.07 \\
\hline
\end{tabular}




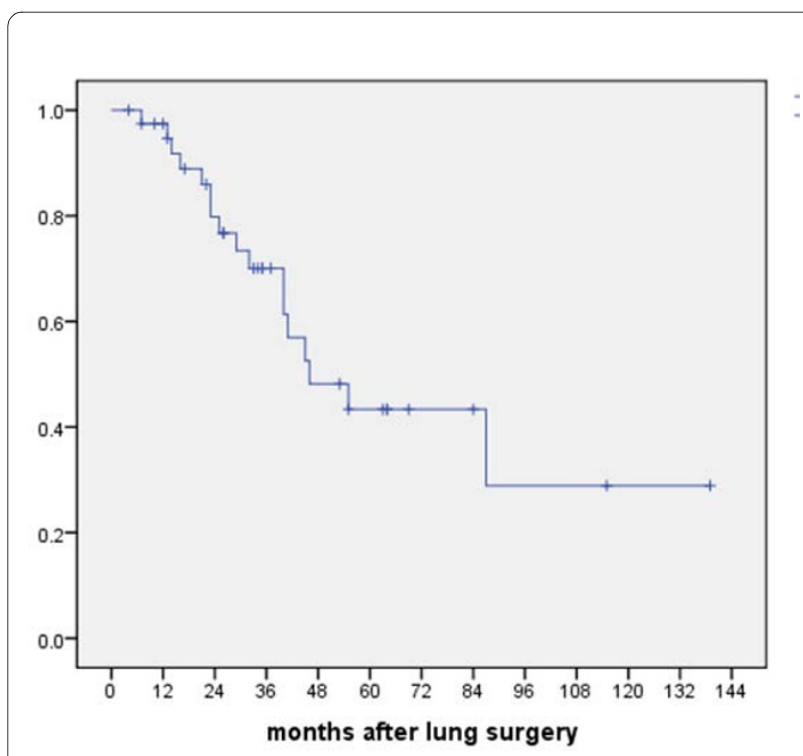

Figure 1 Kaplan-meier overall survival of 40 colorectal cancer patients who underwent resection of lung metastases.

\section{Conclusion}

A minority of CRC patients (10\%) with lung metastases are candidates for surgery with curative intent. In this selected category of patients, a subset has previously developed liver metastases, which were resected with a curative intent. The question of whether to perform pulmonary metastasectomy in this situation will get more complex with advances in chemotherapy. A history of previous liver metastases is certainly not a contra-indication for pulmonary metastasectomy, but the data presented here indicates that these patients have a higher risk of tumor recurrence and a decreased survival in comparison with patients who underwent surgery for

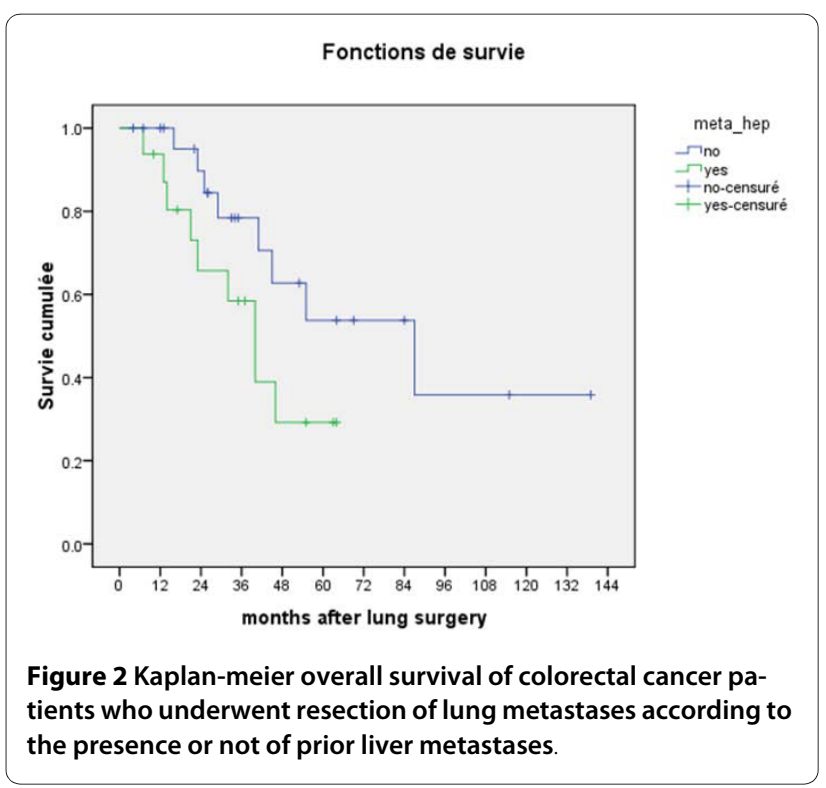

lung-only CRC metastases. These results are clinically relevant, since the incidence of isolated lung metastases without associated liver metastases is low $(<10 \%)$; a history of previously resected liver metastases should be considered a poor prognostic factor in the small subset of CRC patients candidates for pulmonary metastasectomy.

\section{Competing interests}

The authors declare that they have no competing interests.

\section{Authors' contributions}

UL: protocol design, data collection. VO: data collection. JR: protocol design, editing manuscript. GM: editing manuscript. TP: statistical analysis. PM and PG: final review, supervision of scientific content of manuscript. All authors have read and approved the final version of manuscript

\section{Author Details}

'Department of Surgery, Geneva University Hospital and Medical School, Geneva, Switzerland and 2Department of Biostatistics, Geneva University Hospital and Medical School, Geneva, Switzerland

Received: 15 March 2010 Accepted: 3 June 2010

Published: 3 June 2010

\section{References}

1. Andres A, Majno PE, Morel P, Rubbia-Brandt L, Giostra E, Gervaz P, Terraz S, Allal AS, Roth AD, Mentha G: Improved long-term outcome of a Improved long-term outcome of surgery for advanced colorectal liver metastases: reasons and implications for management on the basis severity score. An Surg Oncol 2008, 15:134-43.

2. Ballantyne GH, Quin J: Surgical treatment of liver metastases in patients with colorectal cancer. Cancer 1993, 71:4252-66

3. Fiorentino F, Hunt I, Teoh K, Treasure T, Utley M: Pulmonary metastasectomy in colorectal cancer: a systematic review and quantitative synthesis. JR Soc Med 2010, 103:60-6.

4. Rotolo N, De Monte L, Imperatori A, Dominioni L: Pulmonary resections of single metastases from colorectal cancer. Surg Oncol 2007, 16(Suppl 1):S141-4.

5. Tan KK, de Lima Lopes G, Sim R: How uncommon are isolated lung metastases in colorectal cancer? A review from database of 754 patients over 4 years. J Gastrointest Surg 2009, 13:642-8.

6. Gervaz P, Scholl B, Mainguene C, Poitry S, Gillet M, Wexner S: Angiogenesis of liver metastases: role of sinusoidal endothelial cells. Dis Colon Rectum 2000, 43:980-6.

7. Girard P, Ducreux M, Baldeyrou P, Rougier P, Le Chevalier T, Bougaran J, Lasser P, Gayet B, Ruffié P, Grunenwald D: Surgery for lung metastases from colorectal cancer: analysis of prognostic factors. J Clin Oncol 1996, 14:2047-53

8. Watanabe K, Nagai K, Kobayashi A, Sugito M, Saito N: factors influencing survival after complete resection of pulmonary metastases from colorectal cancer. Br J Surg 2009, 96:1058-65.

9. Pfannschmidt J, Dienemann $\mathrm{H}, \mathrm{Hoffmann} \mathrm{H}$ : Surgical resection of pulmonary metastases from colorectal cancer: a systematic review of published series. Ann Thorac Surg 2007, 84:324-38.

10. Rena O, Casadio C, Viano F, Cristofori R, Ruffini E, Filosso PL, Maggi G: Pulmonary resection for metastases from colorectal cancer: factors influencing prognosis. Twenty-year experience. Eur J Cardiothorac Surg 2002, 21:906-12

11. Takakura Y, Miyata Y, Okajima M, Okada M, Ohdan H: Short disease-free interval is a significant risk factor for intrapulmonary recurrence after resection of pulmonary metastases in colorectal cancer. Colorectal Dis 2009 in press.

12. Lin BR, Chang TC, Lee YC, Lee PH, Chang KJ, Liang JT: Pulmonary resection for colorectal cancer metastases: duration between cancer onset and lung metastasis as an important prognostic factor. Ann Surg Oncol 2009, 16:1026-32.

13. Onaitis MW, Petersen RP, Haney JC, Saltz L, Park B, Flores R, Rizk N, Bains MS, Dycoco J, D'Amico TA, Harpole DH, Kemeny N, Rusch VW, Downey R: Prognostic factors for recurrence after pulmonary resection of colorectal cancer metastases. Ann Thorac Surg 2009, 87:1684-8. 
14. lisaza T, Suzuki M, Yoshida S, Motohashi S, Yasufuku K, lyoda A, Shibuya K, Hiroshima K, Nakatani Y, Fujisawa T: Prediction of prognosis an surgical indications for pulmonary metastasectomy from colorectal cancer. Ann Thorac Surg 2006, 82:254-60.

15. Inoue M, Ohta M, luchi K, Fujiwara K, Fukuhara K, Yasumitsu T: Surgery for pulmonary metastases from colorectal cancer. Ann Thorac Surg 2000, 70:380-3.

16. Pfannschmidt J, Muley T, Dienemann H, Hoffmann H: Prognostic factors and survival after complete resection of pulmonary metastases from colorectal carcinoma: experiences in 167 patients. J Thorac Cardiovasc Surg 2003, 126:732-9.

17. Vogelsang H, Haas S, Hierholzer C, Berger U, Siewert JR, Prauer H: Factors influencing survival after resection of pulmonary metastases from colorectal cancer. Br J Surg 2004, 91:1066-71.

18. Koga R, Yamamoto J, Saiura A, Yamaguchi T, Hata E, Sakamoto M: Surgical resection of pulmonary metastases from colorectal cancer: four favourable prognostic factors. Jpn J Clin Oncol 2006, 36:643-8.

19. Welter S, Jacobs J, Krbek T, Poettgen C, Stamatis G: Prognostic impact of lymph node involvement in pulmonary metastases from colorectal cancer. Eur J Cardiothorac Surg 2007, 31:167-72.

20. Saito Y, Omiya H, Kohno K, Kobayashi T, Itoi K, Teramachi M, Sasaki M, et al.: Pulmonary metastasectomy for 165 patients with colorectal carcinoma. A prognostic assessment. J Thorac Cardiovasc Surg 2002, 124:1007-13.

21. Castaing D, Vibert E, Ricca L, Azoulay D, Adam R, Gayet B: Oncologic results of laparoscopic versus open hepatectomy in two specialized centers. Ann Surg 2009, 250:849-55.

22. Joosten J, Bertholet J, Keemers-Gel M, Barendregt W, Ruers T: Pulmonary resection of colorectal metastases in patients with or without a history of hepatic metastases. Eur J Surg Oncol 2008, 34:895-9.

23. Neeff H, Horth W, Makowiec F, Fischer E, Imdahl A, et al:: Outcome after resection of hepatic and pulmonary metastases of colorectal cancer. $J$ Gastrointest Surg 2009, 13:1813-20.

24. Riquet M, Foucault C, Cazes A, Mitry E, Dujon A, Le Pimpec Barthes F, et al:: Pulmonary resection for metastases of colorectal adenocarcinoma. Ann Thorac Surg 2010, 89:375-80.

25. Yedibela S, Klein P, Feuchter K, Hoffmann M, Meyer T, Papadopoulos T, Gohl J, Hohenberger W: Surgical management of pulmonary metastases from colorectal cancer in 153 patients. Ann Surg Oncol 2006, 13:1538-44

26. Kanemitsu Y, Kato T, Hirai T, Yasui K: Preoperative probability model for predicting overall survival after resection of pulmonary metastases from colorectal cancer. Prognostic factors of pulmonary metastasectomy for colorectal carcinomas. World J Surg 2009, 33:505-11.

\section{Pre-publication history}

The pre-publication history for this paper can be accessed here: http://www.biomedcentral.com/1471-2482/10/17/prepub

\section{Submit your next manuscript to BioMed Central} and take full advantage of:

- Convenient online submission

- Thorough peer review

- No space constraints or color figure charges

- Immediate publication on acceptance

- Inclusion in PubMed, CAS, Scopus and Google Scholar

- Research which is freely available for redistribution

Submit your manuscript at www.biomedcentral.com/submit
C Biomed Central 